\title{
Experimental studies of the vapor phase nucleation of refractory compounds. VI. The condensation of sodium.
}

\author{
Daniel M. Martinez \\ Frank T. Ferguson \\ Richard H. Heist \\ Fairfield University, rheist@fairfield.edu
}

Follow this and additional works at: https://digitalcommons.fairfield.edu/engineering-facultypubs Copyright 2005 American Institute of Physics

The final publisher PDF has been archived here with permission from the copyright holder.

\section{Peer Reviewed}

\section{Repository Citation}

Martinez, Daniel M.; Ferguson, Frank T.; and Heist, Richard H., "Experimental studies of the vapor phase nucleation of refractory compounds. VI. The condensation of sodium." (2005). Engineering Faculty Publications. 143.

https://digitalcommons.fairfield.edu/engineering-facultypubs/143

\section{Published Citation}

Martínez, D. M., Ferguson, F. T., Heist, R. H., \& Nuth III, J. A. (2005). Experimental studies of the vapor phase nucleation of refractory compounds. VI. The condensation of sodium. The Journal of chemical physics, 123(5), 054323. doi:10.1063/1.1998834.

This item has been accepted for inclusion in DigitalCommons@Fairfield by an authorized administrator of DigitalCommons@Fairfield. It is brought to you by DigitalCommons@Fairfield with permission from the rightsholder(s) and is protected by copyright and/or related rights. You are free to use this item in any way that is permitted by the copyright and related rights legislation that applies to your use. For other uses, you need to obtain permission from the rights-holder(s) directly, unless additional rights are indicated by a Creative Commons license in the record and/or on the work itself. For more information, please contact digitalcommons@fairfield.edu. 


\title{
Experimental studies of the vapor phase nucleation of refractory compounds. VI. The condensation of sodium
}

\author{
Daniel M. Martínez ${ }^{\text {a) }}$ \\ Department of Chemical Engineering, University of Rochester, Rochester, New York 14627
}

Frank T. Ferguson

Department of Chemistry, Catholic University of America, Washington, DC 20064

Richard H. Heist

Department of Chemical Engineering, Manhattan College, Riverdale, New York 10471

Joseph A. Nuth III

Code 691, NASA-Goddard Space Flight Center, Greenbelt, Maryland 20771

(Received 19 May 2005; accepted 20 June 2005; published online 10 August 2005)

\begin{abstract}
In this paper we discuss the condensation of sodium vapor and the formation of a sodium aerosol as it occurs in a gas evaporation condensation chamber. A one-dimensional model describing the vapor transport to the vapor/aerosol interface was employed to determine the onset supersaturation, in which we assume the observed location of the interface is coincident with a nucleation rate maximum. We then present and discuss the resulting nucleation onset supersaturation data within the context of nucleation theory based on the liquid droplet model. Nucleation results appear to be consistent with a cesium vapor-to-liquid nucleation study performed in a thermal diffusion cloud chamber. () 2005 American Institute of Physics. [DOI: 10.1063/1.1998834]
\end{abstract}

\section{INTRODUCTION}

Nucleation is the process by which the fragmentary elements of a new phase form within an existing phase, eventually resulting in a phase transition. This process may be carried out homogeneously or heterogeneously depending on system conditions, although homogeneous nucleation has received the bulk of the attention of researchers over the years. ${ }^{1-3}$ In addition, most studies have focused on the vaporto-liquid problem, which has produced a variety of techniques ${ }^{4}$ to obtain data used for testing models that try to describe the homogeneous vapor-to-liquid process.

Nucleation studies typically involve measuring nucleation onset conditions and/or measuring controlled nucleation rates. In certain devices such as the shock tube (ST) ${ }^{5}$ and the expansion cloud chamber (ECC), ${ }^{6}$ the condensable phase is generated within a brief pulse, while in other devices such as the thermal diffusion cloud chamber (TDCC), ${ }^{7}$ the flow diffusion cloud chamber (FDCC), ${ }^{8}$ and the gas evaporation-condensation chamber (GECC), ${ }^{9}$ the condensable phase is generated continuously and fresh vapor must be transported to the nucleation zone. These latter devices (or variants of them) have been used in the development of continuously reinforced aerosol reactors ${ }^{10-14}$ and have received significant experimental and theoretical attention. The GECC has been used for several years to provide nucleation information for metallic and refractory vapors and is the focus of this investigation.

In the evaporation-condensation process associated with these latter devices, vapor is continuously transported from a heated source and cooled in the presence of a background

a)Electronic mail: daniel.m.martinez@ @asa.gov gas, which is then followed by particle formation. In processes that are characterized by large nucleation rates, e.g., the GECC, the nucleated particles introduce sufficient surface area such that particles grow and deplete the vapor. Thus, transport of vapor through a decreasing temperature field will cause a sharp rise in the nucleation rate due to the rapidly increasing supersaturation and the decreasing temperature followed by a sharp reduction in rate due primarily to vapor depletion and latent heat effects associated with particle growth. If particle growth times are fast, then the location and the conditions at the nucleation maximum can be related to the observed vapor particle interface in the experiment and nucleation onset temperature and supersaturation conditions can be calculated.

In this paper we discuss the condensation of sodium vapor and the formation of sodium aerosol occurring in the GECC. A simple one-dimensional model describing vapor transport is employed to determine the onset supersaturation, in which we assume the observed location of the vapor/ aerosol interface is associated with a nucleation rate maximum. We then present and discuss the resulting nucleation onset supersaturation data within the context of conventional nucleation models and compare our results with other alkali metal nucleation data obtained using the TDCC.

\section{THE GAS EVAPORATION-CONDENSATION CHAMBER}

\section{A. Background}

The inert gas evaporation-condensation technique has been in use for nearly seventy years and is a proven method for generating particles of small sizes and with narrow size distributions. ${ }^{10-12}$ The technique takes full advantage of the 


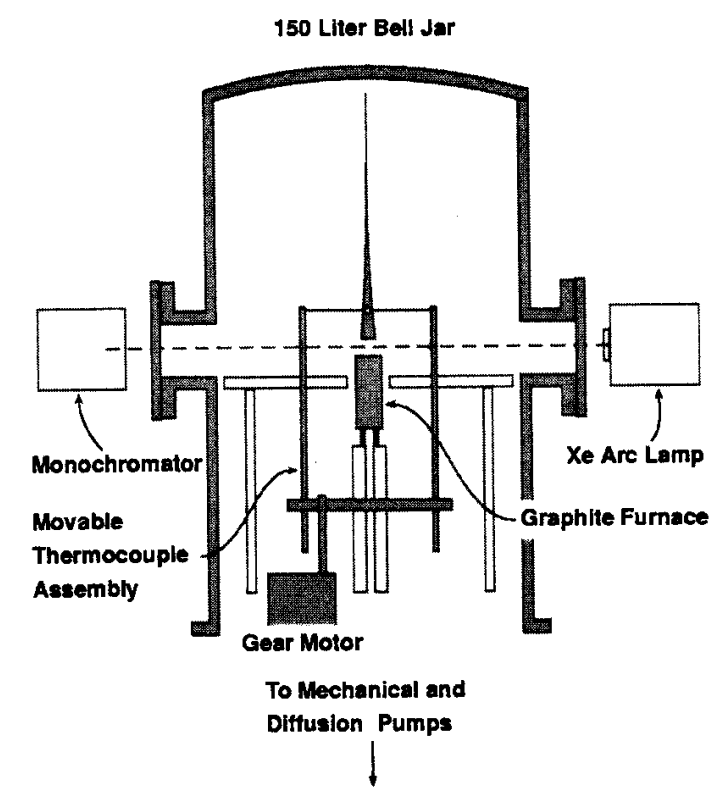

FIG. 1. Cross-sectional diagram of the gas evaporation condensation chamber.

nucleation process, whereby heat from a diffusing metal vapor is transferred to the surrounding cooler, inert background gas, creating a supersaturation large enough to initiate a nucleation event. The original focus was on determining correlations between particle size and experimental conditions, as well as understanding the growth process of particle production. More recently, this technique is being utilized to conduct quantitative nucleation research. 15,16

The primary benefits of using a GECC for nucleation investigations over other devices are the relative ease of operation (especially with respect to metallic species) and the ability to measure directly the temperature field within the experimental chamber. A drawback of the method is that unless conditions can be accurately measured at the interface, the concentration of the diffusing material from the vapor source to the nucleation zone must be calculated as in a TDCC, for example. Also, buoyancy induced convection plays a larger role in determining the overall flow of the condensing vapor and background gas. ${ }^{17}$

\section{B. Description}

The GECC consists of a stainless steel crucible resistively heated by a graphite furnace. The crucible/furnace assembly is contained within a 150-liter stainless steel bell jar that is jacketed with copper piping to cool the chamber if necessary. The jar is equipped with four view ports that permit optical access. For viewing, the inside of the chamber can be illuminated using a xenon arc lamp. A video camera oriented 90 degrees to the arc lamp beam is used to record the experiments. The system is connected to a gas handling manifold with an inert gas supply and a set of mechanical and diffusion pumps that are capable of controlling the internal pressure over a range from $10^{-4}$ to approximately $10^{5} \mathrm{~Pa}$. A schematic of the experimental setup is provided in Fig. 1.

The temperature profile along the centerline of the chamber (from the crucible containing the liquid sodium to the nucleation zone) is measured during an experiment using a movable thermocouple assembly. This assembly consists of two stainless steel rods attached to a movable stage. This assembly is also used to track any movement of the vapor/ aerosol interface. A thin-wire, uninsulated thermocouple is stretched between the two leads in such a fashion that the junction is oriented directly over the center of the crucible. We point out that temperature measurements made in the presence of the condensing material are made after the interface is fully developed. To verify that the method of temperature measurement has no effect on the condensation process, temperature readings are taken both in the absence and presence of condensing material. We have observed no differences in results obtained in either fashion. In addition, we observe that the shape and location of the nucleation interface is unaffected by the movement of the thermocouple wire.

\section{A typical experiment}

During a typical evaporation/condensation experiment, the crucible is filled with sodium metal and the chamber closed. The chamber is then evacuated and filled with an inert background gas. The graphite furnace surrounding the crucible is then used to heat the metal in the crucible. A thin layer of ceramic material is placed around the crucible to prevent direct contact with the graphite. When sufficiently heated, vapor rises from the furnace-crucible assembly and after a short distance (e.g., one to two centimeters) forms a buoyant plume of smoke particles directly above the liquid metal surface. It is important to note that there is a sharp, distinct interface between the region that contains vapor rising from the liquid surface and the particle-rich region of the smoke plume. In our analysis, we take this sharp interface to be the location of the nucleation front.

In our experiments with sodium metal, we observe a faint, but visible, orange-red glow extending axially (vertically) above the furnance opening during the initial stages of an experiment. This is due to the fluorescence of the sodium vapor under illumination from the high-pressure xenon arc lamp. As heating continues and the temperature increases, the glow becomes more intense and extends farther away (vertically) from the furnace opening. As we continue heating the sample, a green glow appears directly above the top of the furnace opening, which we attribute to fluorescence of the sodium dimer. The dimer concentration is small (roughly $1 \%$ under the conditions used in this investigation) but the fluorescence is sufficiently intense that it obscures the fluorescence of the sodium atoms. The observation of the monomer and dimer fluorescence is important because it helps us visualize the flow geometry of the vapor-gas mixture as it is transported to the nucleation zone. Since we can distinguish the sharp nucleation interface between the vapor and the newly forming aerosol we are able to identify the two important distinct regions (e.g., vapor-gas transport region and nucleation-aerosol growth region) used in our modeling of the transport and nucleation processes occurring in the GECC.

Once the vapor/aerosol interface is fully developed, we record the temperature profile along the centerline of the 
GECC and make an optical absorption measurement at the furnace opening to determine the vapor concentration at that location. We do this because as the sodium metal melts, the height of the molten liquid pool drops below the opening of the crucible and we lose access to measuring its location and its temperature during an experiment. Moreover, due to physical constraints, we are limited to taking this spectroscopic measurement at one fixed location, prohibiting reliable measurement of the vapor concentration at the particle interface, which changes location when the heater temperature is changed. Thus, we take a measurement and then use a model for the vapor transport to determine the concentration at the interface location. Details of this optical absorption measurement are given elsewhere. ${ }^{16}$

Finally, we make an optical extinction measurement at the nucleation zone in order to measure the aerosol particle number density and estimate a nucleation rate. The same system as used for the absorption measurements is used for the extinction measurement, however, the furnace assembly is moved down slightly so that the optical path passes through the condensed sodium particulates. The attenuation of the light intensity, $I$, by the smoke particles at condensation temperatures of the experiment is measured over the wavelengths, $\lambda$, of $350-650 \mathrm{~nm}$. The unattenuated intensity, $I_{0}$, is also measured at the same temperatures with no particles present. Finally, the path length, $L$, over which extinction occurs is assumed to be equal to the width of the smoke plume at that location, measured from digitized video images of the plume during the run.

The particle size, $d_{p}$, and particle number density, $N$, can then be determined by using the Beer-Lambert law:

$$
\frac{I}{I_{0}}=\exp (-\gamma L)
$$

where $\gamma$ is the extinction coefficient. Assuming a monodisperse system of spherical particles, the extinction coefficient at any wavelength is given by

$$
\gamma(\lambda)=N \pi \frac{d_{p}^{2}}{4} Q_{e x t}\left(d_{p}, \lambda, m\right),
$$

where $Q_{e x t}$ is the extinction efficiency factor for a particular particle diameter, wavelength and refractive index $m$. Data for the wavelength refractive indexes for sodium were taken from Ref. 18. This efficiency factor is calculated using Mie scattering theory. We select a particle size and number density to calculate $Q_{e x t}$ and then use the Beer-Lambert law to calculate the intensity ratio. We arrive at a final solution when the selection of a combination of particle size and number concentration minimizes the error between the law and what we measure in the extinction measurement over the aforementioned range of wavelengths.

\section{Vapor transport model}

Along with the measured centerline temperature profile, the vapor transport within our experimental setup is modeled using the following equation:
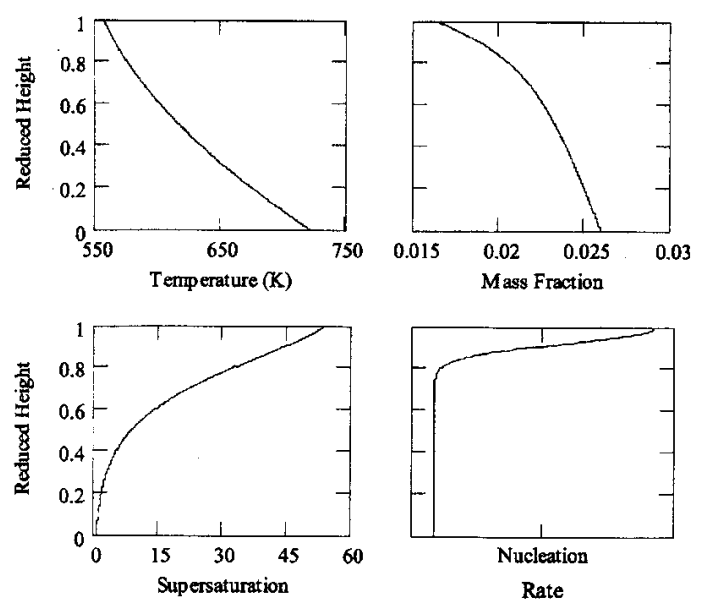

FIG. 2. Typical chamber profiles of temperature, mass fraction, supersaturation, and nucleation rate as a function of reduced height from near the crucible opening up to the measured vapor/aerosol interface.

$$
\frac{d w_{A}}{d z}=\frac{1}{\rho_{g} \nu}\left[\frac{d}{d z}\left(\rho_{g} D_{A B} \frac{d w_{A}}{d z}\right)\right]
$$

where $w_{A}$ is the mass fraction of the condensing species, $z$ is the centerline position above the crucible, $\rho_{g}$ is the total gas density, $\nu$ is the local fluid velocity (vapor plus background gas) along the centerline of the GECC (based on a twodimensional numerical solution described in Refs. 19 and 20 that varies with source furnace temperature), and $D_{A B}$ is the binary diffusion coefficient. In this model, radial dispersion is ignored.

In order to define the upper boundary (nucleation zone) to solve Eq. (3), we assume that the location of the particle interface is coincident with a maximum in nucleation rate. This is consistent with observations made using the FDCC where it was determined that the predicted nucleation rate maximum coincided with the location in the chamber of droplet formation. ${ }^{8}$ In operation, the FDCC is similar to the GECC, except that in the GECC the flow component occurs naturally and the nucleation rate at the vapor/aerosol interface is substantially greater. Additionally, in devices that are characterized by large nucleation rates, such as the supersonic nozzle (SN), ${ }^{21}$ the nucleation zone is detected by the particles that grow subsequent to the initial nucleation event.

Thus, the boundary conditions used to solve Eq. (1) are as follows: (1) at the location of the optical concentration measurement the mass fraction of the sodium vapor is taken to be equal to that obtained from the optical measurement; (2) at the vapor/aerosol interface, the mass fraction is equal to that value that will maximize the nucleation rate, $J$, as determined using an appropriate nucleation rate expression (i.e., $d J / d z=0$ at the interface). The expression for the nucleation rate used in this analysis was based on the classical nucleation theory (CNT) expression: ${ }^{22,23}$

$$
J=\left(\frac{2 \sigma v_{A}^{2}}{\pi m_{A}}\right)^{1 / 2}\left(\frac{S P_{v a p}}{k_{B} T}\right)^{2} \exp \left(-\frac{16 \pi \sigma^{3} v_{A}^{2}}{3 k_{B}^{3} T^{3} \ln ^{2} S}\right),
$$

where $\sigma$ is the surface tension, $v_{A}$ is the molecular volume, $m_{A}$ is the molecular mass, $S$ is the supersaturation ratio, $k_{B}$ is Boltzmann's constant, $T$ is temperature, and $P_{v a p}$ is the equi- 
TABLE I. Thermophysical properties of sodium and helium. Values of molecular weight, $M(\mathrm{~g} / \mathrm{mol})$; liquid density, $\rho\left(\mathrm{g} / \mathrm{cm}^{3}\right)$; surface tension, $\sigma$ (dyne/cm); equilibrium vapor pressure, $P_{v a p}(\mathrm{~mm} \mathrm{Hg})$; binary diffusion coefficient, $D_{12}\left(\mathrm{~cm}^{2} / \mathrm{s}\right) . T$ is the temperature in $\mathrm{K}$.

$$
\begin{aligned}
& \text { Sodium } \\
& \qquad \begin{array}{l}
M=22.99 \\
\quad=0.927-0.000235(T-371)^{\mathrm{a}} \\
\sigma=202-0.1(T-371)^{\mathrm{b}} \\
\log P_{\text {vap }}=10.86423-5619.406 T^{-1}+0.00000345 T \\
-1.0411 \log T^{\mathrm{c}}
\end{array} \\
& \text { Helium } \\
& \quad M=4.0026 \\
& D_{12}(\mathrm{Na}-\mathrm{He})=2.5\left(98.1 / P_{\text {tot }}\right)(T / 723)^{1.65 \mathrm{~d}}
\end{aligned}
$$

Reference 18 .

${ }^{\mathrm{b}}$ Reference 32.

${ }^{\mathrm{c}}$ Reference 33.

${ }^{\mathrm{d}}$ Reference 34 .

librium vapor pressure. Typical temperature, mass fraction, supersaturation, and nucleation rate profiles as a function of reduced vertical height (up to the interface) using Eqs. (3) and (4) are shown in Fig. 2. Thermophysical properties used to calculate these profiles (except temperature) and theoretical nucleation rates are provided in Table I.

We stress that the purpose of employing a model nucleation expression is not to derive a quantitative value for rate, but rather the value of the concentration at the interface when this expression is maximized. Thus, any reasonable nucleation rate expression that exhibits the usual strong dependence on supersaturation and temperature can be used in this analysis and will give, to a good approximation, the same value for supersaturation at the interface. In fact, we determined that supersaturation values determined by the massaction consistent classical theory (MACCT ${ }^{24}$ or the internally consistent classical theory (ICCT) ${ }^{25,26}$ fall within $2 \%$ of the predictions obtained using Eq. (4).

Finally we note that although we are confident that the nucleation onset model we use portrays an accurate description of the evaporation/condensation process in our GECC up until the interface location, we expect that the onset supersaturations we calculate can be larger than if we explicitly accounted for growth in our model. We do not, however, expect this difference to be large because by saying that a nucleation rate maximum occurs at the vapor/aerosol interface we are saying that nucleation must die down rapidly due to downstream (i.e., toward the top of the bell jar) effects such as particle growth. We would be more concerned with growth's impact if the physical problem were similar, for example, to the TDCC study of various alcohol vapors at high nucleation rates. ${ }^{27}$ There, the growing particles are known to re-enter the vapor region, thus stripping the diffusing vapor and causing a substantial depletion of the vapor concentration. In our situation, the vapor is continually reinforced and particles do not re-enter the vapor-rich region.

\section{RESULTS}

We report nucleation onset measurements made on sodium vapor. The experimental data are listed in Table II. In Table II the experimental run number is listed first followed by centerline temperature measurements and positions at experimentally important locations, followed by partial pressures near the furnace opening determined from the spectroscopic measurement. Finally, the supersaturations at onset, calculated as described above, are presented in the last column. The experiments were conducted at an ambient pressure of $20 \mathrm{kPa}$ of helium. During our experiments, we observed that at higher furnace source temperatures, the vapor/ aerosol interface appeared at a location closer to the furnace opening. Typical of onset experiments, the supersaturation decreases with increasing temperature.

Based on the procedure described earlier for measuring particle density, we measure the number density in the immediate region of the vapor/aerosol interface to be roughly constant for the experiment and approximately equal to $2 \times 10^{7 \pm 1}$ particles per cubic centimeter. Significant differences in the particle density of the smoke plume over the ninety-degree temperature range of the experiment were not detectable, supporting this result. We then estimate the nucleation rate to be $\sim 3 \times 10^{9 \pm 1}$ particles per cubic centimeter per second for the experiment using a local fluid velocity at the vapor/aerosol interface of 30 centimeters per second, and a nucleation zone thickness of 2 millimeters.

TABLE II. Experimental data obtained from the condensation of sodium vapor in a helium background gas. The first column is the experiment number; the next three are the vapor/aerosol interface temperature, $T_{\text {int }}$, the crucible/furnace opening temperature, $T_{\text {top }}$, and the spectroscopic location temperature, $T_{\text {spec }}$, respectively. The fifth and sixth columns are the vapor/aerosol interface location, $z_{\text {int }}$, and the spectroscopic measurement location, $z_{\text {spec }}$, respectively. The seventh column represents the vapor partial pressure obtained at the spectroscopic location, $P_{\text {spec }}$. The last column is the supersaturation calculated at the vapor/aerosol interface, $S_{\text {onset }}$. Experiments were conducted at a total pressure of $20 \mathrm{kPa}$.

\begin{tabular}{cccccccc}
\hline \hline Run & $T_{\text {int }}(\mathrm{K})$ & $T_{\text {top }}(\mathrm{K})$ & $T_{\text {spec }}(\mathrm{K})$ & $z_{\text {int }}(\mathrm{cm})$ & $z_{\text {spec }}(\mathrm{cm})$ & $P_{\text {spec }}(\mathrm{kPa})$ & $S_{\text {onset }}$ \\
\hline 32 & 556.15 & 721.15 & 668.15 & 1.44 & 0.33 & 0.08604 & 53.88 \\
45 & 566.15 & 742.15 & 686.15 & 1.43 & 0.33 & 0.12253 & 49.20 \\
59 & 575.15 & 751.15 & 695.15 & 1.41 & 0.33 & 0.16851 & 47.86 \\
17 & 606.15 & 783.15 & 720.15 & 1.34 & 0.33 & 0.33699 & 34.03 \\
14 & 615.15 & 792.15 & 728.15 & 1.34 & 0.33 & 0.39213 & 28.00 \\
39 & 627.15 & 810.15 & 739.15 & 1.26 & 0.33 & 0.57004 & 30.52 \\
11 & 646.15 & 820.15 & 758.15 & 1.18 & 0.33 & 0.77869 & 21.09 \\
\hline \hline
\end{tabular}




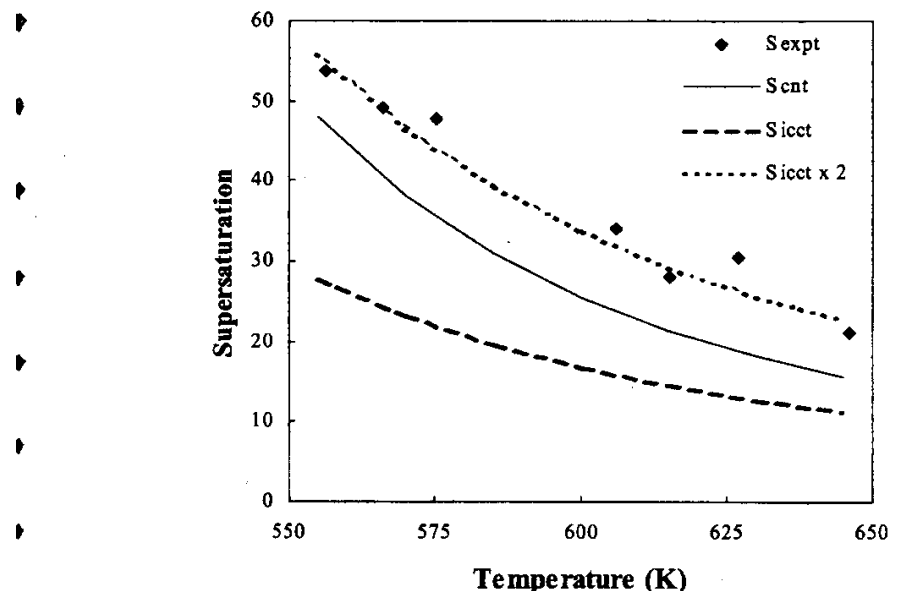

FIG. 3. Comparison of the nucleation onset data (solid diamonds) with the classical nucleation theory (solid curve) and the internally consistent classical theory (dashed curve) for a nucleation rate of $3 \times 10^{9} \mathrm{~cm}^{-3} \mathrm{~s}^{-1}$. The temperature dependence of the supersaturation data closely resembles that of the internally consistent theory (dotted curve).

\section{COMPARISON TO THEORY}

We compare the supersaturation onset data with the CNT and ICCT predictions for sodium at a nucleation rate of $3 \times 10^{9} \mathrm{~cm}^{-3} \mathrm{~s}^{-1}$ in Fig. 3. We see that the two model predictions lie below the experimental data. The temperature dependence of the onset supersaturation data, however, compares rather well with the predictions of the ICCT. In fact, when we arbitrarily multiply the supersaturation data calculated using the ICCT by a factor of 2 , the agreement with the experimentally obtained onset data is good. A curve fitted to these data demonstrating this is also displayed in Fig. 3.

The observation that the ICCT underpredicts our experimental results by a factor of 2 may have relevance with respect to the nucleation behavior of alkali metals in general since this same observation was made by investigators in a cesium vapor nucleation study using a TDCC. ${ }^{28}$ However, more experimental work with the other alkali metals should be performed to verify this comparison to theory, since it has been shown elsewhere ${ }^{29}$ that the internally consistent modification to the classical model is an arbitrary and unfounded method for handling a truncation error. Furthermore, it has been demonstrated that electronic magic numbers can play a role in the nucleation behavior of the alkali metals, ${ }^{30}$ which are not explicitly accounted for in the classical models. Sodium and cesium metals have been shown experimentally to have identical sizes of magic numbers, which may help explain their similarity in nucleation behavior with respect to the classical model and its modifications.

\section{CONCLUSIONS}

The homogeneous nucleation of sodium vapor was studied in a gas evaporation condensation chamber. Onedimensional transport of the condensing vapor was modeled up to the vapor/aerosol interface where it was assumed that the nucleation rate was a maximum. The supersaturation at nucleation onset was then determined using measured experimental information and finally compared to conventional nucleation models.
Sodium vapor condensation data obtained over a temperature range from $555 \mathrm{~K}$ to $646 \mathrm{~K}$ yielded nucleation onset supersaturation ratios of $\sim 54$ to $\sim 21$, respectively, at an estimated nucleation rate of $3 \times 10^{9} \mathrm{~cm}^{-3} \mathrm{~s}^{-1}$. It was noted that the temperature dependence of measured onset supersaturation was similar to the prediction of the internally consistent classical theory and that the supersaturation values differed numerically from the predictions of this modification by a factor of 2. Also, the critical cluster sizes, calculated using the experimental supersaturations and the Kelvin equation, range from 13 atoms/cluster at $556 \mathrm{~K}$ to 16 atoms/cluster at $646 \mathrm{~K}$. These results are consistent with results from a cesium nucleation study performed using a TDCC, ${ }^{28}$ thus providing experimental evidence for similar nucleation behavior between two alkali metals in two different experimental devices.

Further experimental work will need to be performed in order to explore whether this observed nucleation behavior extends to the rest of the alkali metals. Although it has been demonstrated elsewhere that the internally consistent modification to the classical nucleation theory has no true theoretical basis, ${ }^{29}$ it is curious that the temperature dependence on supersaturation it predicts is similar to a wide range of materials, from organic liquids such as the n-alkanes ${ }^{31}$ to, at least, two of the alkali metals. Continued work is necessary to rectify the apparent problems that exist with the theoretical underpinnings of the classical model.

${ }^{1}$ A. C. Zettlemoyer, Nucleation (Dekker, New York, 1969).

${ }^{2}$ P. G. Debenedetti, Metastable Liquids (Princeton, New Jersey, 1996).

${ }^{3}$ G. S. Springer, Advances in Heat Transfer (Academic, New York, 1978), Vol. 14.

${ }^{4}$ R. H. Heist and H. H. He, J. Phys. Chem. Ref. Data 23, 781 (1994).

${ }^{5}$ J. R. Stephens and S. H. Bauer, Proceedings of the 13th International Symposium on Shock Tubes and Waves (State University of New York Press, Albany, NY, 1982).

${ }^{6}$ J. L. Schmitt, Metall. Trans. A 23, 1957 (1992).

${ }^{7}$ R. H. Heist and H. Reiss, J. Chem. Phys. 59, 665 (1973).

${ }^{8}$ V. Vohra and R. H. Heist, J. Chem. Phys. 104, 382 (1996).

${ }^{9}$ J. A. Nuth III and B. Donn, J. Chem. Phys. 77, 2639 (1982).

${ }^{10}$ S. Yatsuya, S. Kasukabe, and R. Uyeda, Jpn. J. Appl. Phys. 12, 1675 (1973).

${ }^{11}$ C. G. Granqvist and R. A. Buhrman, J. Appl. Phys. 47, 2200 (1976).

${ }^{12}$ S. G. Kim and J. R. Brock, J. Appl. Phys. 60, 509 (1986).

${ }^{13}$ D. R. Warren and J. H. Seinfeld, Aerosol Sci. Technol. 3, 135 (1984).

${ }^{14}$ H. He, R. H. Heist, B. L. MeIntire, and T. N. Blanton, Nanostruct. Mater. 8, 879 (1997).

${ }^{15}$ F. T. Ferguson, J. A. Nuth III, and L. U. Lilleleht, J. Chem. Phys. 104, 3205 (1996).

${ }^{16} \mathrm{~F}$. T. Ferguson and J. A. Nuth III, J. Chem. Phys. 113, 4093 (2000).

${ }^{17}$ B. N. Hale, P. Kemper, and J. A. Nuth III, J. Chem. Phys. 91, 4314 (1989).

${ }^{18} \mathrm{CRC}$ Handbook of Chemistry and Physics, 78th ed. (CRC, New York, 1997).

${ }^{19}$ S. Patankar, Numerical Heat Transfer and Fluid Flow (Hemisphere, Washington, D.C., 1980).

${ }^{20}$ F. T. Ferguson, "Numerical simulation of natural convective flows within a nucleation chamber under variable gravity," Ph.D. thesis, University of Virginia, Charlottesville, Virginia (1993).

${ }^{21}$ B. E. Wyslouzil, G. Wilemski, M. G. Beals, and M. B. Frish, Phys. Fluids 6, 2845 (1994).

${ }^{22}$ R. Becker and W. Doring, Ann. Phys. 24, 719 (1935).

${ }^{23}$ J. Zeldovich, J. Exp. Theor. Phys. 12, 525 (1942).

${ }^{24}$ W. G. Courtney, J. Chem. Phys, 35, 2249 (1961).

${ }^{25}$ M. Blander and J. L. Katz, J. Stat. Phys. 4, 55 (1972).

${ }^{26}$ S. L. Girshick and C.-P. Chiu, J. Chem. Phys. 93, 1273 (1990).

${ }^{27}$ A. Kacker and R. H. Heist, J. Chem. Phys. 82, 2734 (1985). 
${ }^{28}$ M. M. Rudek, J. L. Katz, and H. Uchtmann, J. Chem. Phys. 110, 11505 (1999).

${ }^{29}$ R. B. McClurg and R. C. Flagan, J. Colloid Interface Sci. 201, 194 (1998).

${ }^{30}$ R. Bahadur and R. B. McClurg, J. Chem. Phys. 121, 12499 (2004).

${ }^{31}$ M. M. Rudek, J. A. Fisk, and J. L. Katz, J. Chem. Phys. 105, 4707 (1996)
${ }^{32}$ C. C. Addison, W. E. Addison, D. H. Kerridge, and J. Lewis, J. Chem. Soc. 1955, 2202 (1955).

${ }^{33}$ A. N. Nesmayanov, Vapor Pressure of the Elements (Academic, New York, 1963).

${ }^{34}$ K. M. Aref'ev, V. M. Borishanskii, L. A. Vorontsova, T. V. Zablotskaya, N. I. Ivashchenko, I. I. Paleev, and B. M. Khomchenkov, Teplofiz. Vys. Temp. 10, 1112 (1972). 